\title{
Ultra-Sensitive Elemental and Isotope Measurements with Compact Plasma Source Cavity Ring-Down Spectroscopy (CPS-CRDS)
}

PI: Yixiang Duan, C-ACS, K484, Los Alamos National Laboratory, Los Alamos, NM, 87545

Co-PI: Chuji Wang, Diagnostic Instrumentation and Analysis Laboratory, 205 Research Boulevard, Starkville, Mississippi State University, MS 39759

Co-PI: Christopher B. Winstead, Department of Physics and Astronomy, University of Southern Mississippi, USM Box 5046, Hattiesburg, Mississippi, 39406

\section{Research Objective}

The proposed research is to develop a new class of instruments for actinide isotopes and hazardous element analysis through coupling highly sensitive cavity ring-down spectroscopy to a compact microwave plasma source. The research work will combine advantages of CRDS measurement with a low power, low flow rate, tubing-type microwave plasma source to reach breakthrough sensitivity for elemental analysis and unique capability of isotope measurement. The project has several primary goals: 1). Explore the feasibility of marrying CRDS with a new microwave plasma source; 2). Provide quantitative evaluation of CMP-CRDS for ultratrace elemental and actinide isotope analysis; 3). Approach a breakthrough detection limit of ca. $10^{-13} \mathrm{~g} / \mathrm{ml}$ or so, which are orders of magnitude better than currently available best values; 4). Demonstrate the capability of CMP-CRDS technology for isobaric measurements, such as ${ }^{238} \mathrm{U}$ and ${ }^{238} \mathrm{Pu}$ isotopes. 5). Design and assemble the first compact, field portable CMP-CRDS instrument with a high-resolution diode laser for DOE/EM on-site demonstration. With all these unique capabilities and sensitivities, we expect CMPCRDS will bring a revolutionary change in instrument design and development, and will have great impact and play critical roles in supporting DOE's missions in environmental remediation, environmental emission control, waste management and characterization, and decontamination and decommissioning. The ultimate goals of the proposed project are to contribute to environmental management activities that would decrease risk for the public and workers, increase worker productivity with on-site analysis, and tremendously reduce DOE/EM operating costs.

\section{Research Progress and Implications}

This report summarizes work from 09/15/2002 to 05/31/2003 of a three-year project. Major achievements are attributed to the following five aspects described below. Due to the limited length of the report, plenty of experimental data and figures are presented in Optional Additional Information in http://emsp.osti.gov.

(1) Demonstrated the feasibility of combining CRDS with compact microwave plasma $(C M P)$ source: "combination of microwave plasma with CRDS is a very interesting and original research area" as one of the paper reviewers commented. Any one of factors in the system such as plasma power, gas flow rates, and measuring locations 
affects the system performance, e.g. ringdown baseline stability. We used laboratory CRDS system consisting of a pulsed tunable dye laser to test and build a science base and to demonstrate the feasibility of the new instrument. A laboratory designed and built sampling system for solution sample introduction was used for the new instrument testing. The ringdown signals were monitored using a photomultiplier tube and recorded using a digital oscilloscope interfaced to a computer. Lead was chosen as a typical element for the system optimization and characterization. Baseline noise of the plasma source has been thoroughly studied in this work. A detection limit of $0.8 \mathrm{ppb}\left(10^{-10}\right)$ is obtained with such a device.

(2) Successfully introduced a compact diode laser as the light source thus formed a compact laser and compact plasma CRDS system: One of essential steps in the research project is to replace the pulsed laser system with a compact continuous wave $(\mathrm{CW})$ diode laser. A single frequency, compact, external diode laser was successfully introduced to the system; correspondingly, new optical configurations and electronic control were developed. The ringdown baseline noise $\left(\sigma / \tau_{\text {(average) }}\right)$ was obtained to be $0.2 \%$. This important progress has promoted the laboratory research towards the instrumentation.

(3) Conducted extensive research with multiple orientations for direct comparison with different plasmas and laser sources: Multiple laser sources (pulsed and $\mathrm{CW}$ ) were used in combination with two atomization/ionization sources, a low power inductively coupled plasma (ICP) and a compact microwave induced plasma. Strontium ( $\mathrm{Sr}$ ) was selected as a solution sample. The plasma-generated $\mathrm{SrOH}$ radical has a red narrow band transition around $680 \mathrm{~nm}$, where both the pulse laser and the diode laser have the wavelength output and a uranium red line $(682 \mathrm{~nm})$ is also located. In order to directly compare and contrast the individual systems, multiple configurations have been explored. Results obtained when one laser source was operated with each plasma source (ICP and CMP) are compared as are results obtained with one plasma source and both lasers. Detection sensitivity, system optimization, ringdown baseline noise, as well as potential improvements, are discussed.

(4) Compact and tubing-shaped plasma sources with several different geometries were designed, fabricated, and scheduled for characterizations: Initial research was started with a microwave plasma source called microwave plasma torch (MPT), which was used as an atomization cell for ring-down measurement. The advantages of the MPT plasma source include low power, low gas flow rate, extremely stable, and ease of handle. However, since the MPT plasma takes a toroidal shape, it has limitation in absorption path length. To overcome this limitation and enhance the system performance, we have being working on designing a tubing-shaped plasma source with various geometries. Currently our work is emphases on the discharge tube design, optimization of the tube diameter, and test different geometries in order to get better performance. These tubing-shaped plasma discharges will be coupled with cavity ring-down measurement in the next step and to examine the system performance.

(5) Scientific contribution: In the R \& D of a plasma-CRDS directed to instrumentation, another important benefit is the generation of abundant and original scientific 
information about plasma temperature profile, electron density distribution, thermodynamic behavior, $\mathrm{OH}$ radical density profile. These scientific data not only enriches the plasma science, but also provides important information for atmospheric plasma application and high temperature combustion diagnostics.

\section{Planned Activities}

In this reported period, significant progresses are made and research activities are on schedule. In the next fiscal year, following research activities are planned:

- Finish the design and testing of the new tube-shaped plasma source $\left(1^{\text {st }}-3^{\text {rd }}\right.$ month).

- Obtain emission spectra of multiple elements from newly designed compact plasma and diagnose chemical and physical properties of new plasma sources. These researches will help to build a science base for plasma performances and ringdown absorption measurements ( $1^{\text {st }}-3^{\text {rd }}$ month).

- Ringdown measurements of several elements and isotopes using various plasma sources at different wavelength regions $\left(4^{\text {th }}-9^{\text {th }}\right.$ month $)$.

- Upgrade instrument configurations toward compactness and robustness, e.g. introducing optical fiber as waveguide to replace optical lens system $\left(10^{\text {th }}-12^{\text {th }}\right)$.

\section{Information Access:}

- Exploration of Microwave Plasma Source Cavity Ring-Down Spectroscopy for Highly Sensitive Elemental Measurements, Yixiang Duan, Chuji Wang, and Christopher B. Winstead, Anal. Chem. 75, 2105 (2003).

- Isotopic Measurements Of Uranium Atom/Ion Using Cavity Ringdown Spectroscopy, Chuji Wang, F. J. Mazzotti, G. P. Miller, and C. B. Winstead, Appl. Spectrosco. (To be in 57 (9), 2003).

- Elemental and Isotopic Measures Using Plasma Diode Laser Cavity Ringdown Spectroscopy, Sudip P. Koirala, S. T. Scherrer, Chuji Wang, Yixiang Duan, and Christopher B. Winstead, Submitted in FACSS Oct. 2003.

- Elemental Analysis Using Compact Plasma-Cavity Ringdown Spectroscopy with Diode Laser, Sudip P. Koirala, S. T. Scherrer, Chuji Wang, Yixiang Duan, and Christopher B. Winstead, (in preparation).

\section{Optional Additional Information}

(Submitted in http://emsp.osti.gov)

\section{Optional Proprietary Information}

- Method and Apparatus for Elemental and Isotope Measurements and DiagnosticsMicrowave Induced Plasma-Cavity Ring-Down Spectroscopy, Chuji Wang, Christopher B Winstead, and Yixiang Duan, Patent pending \# 10/367,806. 